\title{
Outcome of cephalic vein cut-down approach: A safe and feasible approach for totally implantable venous access device placement
}

\author{
SHINICHIRO KOKETSU ${ }^{1}$, SHINISHI SAMESIMA ${ }^{1}$, SATOMI YONEYAMA $^{2}$, TOSHIYUKI OKADA ${ }^{2}$, \\ SHIGERU TOMOZAWA ${ }^{2}$, HIROYUKI HORIKOSHI ${ }^{3}$ and TOSHIO SAWADA ${ }^{2}$ \\ ${ }^{1}$ First Department of Surgery, Koshigaya Hospital, Dokkyo Medical University, Saitama; \\ Departments of ${ }^{2}$ Surgery, and ${ }^{3}$ Radiology, Gunma Cancer Center, Gunma, Japan
}

Received July 12, 2010; Accepted September 11, 2010

DOI: 10.3892/ol.2010.189

\begin{abstract}
This study aimed to evaluate the safety and feasibility of the venous access via the cephalic vein cut-down (CVCD) approach for totally implantable venous access device (TIVAD) placements. A total of 79 patients who received TIVAD for the treatment of unresectable or recurrent colorectal carcinomas were recruited. The operation time and the complications were evaluated. Results showed the TIVAD placement via the CVCD approach was successful in 74 patients. A total of 5 patients required conversion to a percutaneous puncture approach. The mean operation time was $34.7 \mathrm{~min}$. No intraoperative or postoperative complications were observed. Therefore, the CVCD approach is a safe and feasible method for TIVAD placement.
\end{abstract}

\section{Introduction}

Significant improvements have been made in the chemotherapy of unresectable or recurrent colorectal carcinomas. These improvements are due to the development of combination chemotherapies, including fluorouracil, irinotecan or oxaliplatin, in combination with molecular-targeting agents, including bevacizumab and cetuximab. The use of a totally implantable venous access device (TIVAD) is recommended for administration of these chemotherapeutic agents.

TIVADs are generally placed in position by the percutaneous subclavian vein approach. This approach causes infrequent intraoperative or postoperative complications, including pneumothorax, arterial puncture, hemothorax, injury to brachial plexus (1-5) and pinch-off syndrome (6).

Venous access via the cephalic vein cut-down (CVCD) method has been widely described as a safe and rapid approach (7-10). However, this technique is not currently widely used

Correspondence to: Dr Shinichiro Koketsu, First Department of Surgery, Koshigaya Hospital, Dokkyo Medical University, 2-1-50 Minamikoshigaya, Koshigaya, Saitama 343-8555, Japan E-mail: koketsu-tky@umin.ac.jp

Key words: totally implantable venous access device, cut-down, cephalic vein, chemotherapy, colorectal cancer for the placement of TIVADs. Since the cephalic vein shows few anatomical anomalies (11), this approach is suitable for the placement of TIVADs.

This study examined the outcome of the venous access via the CVCD method for the placement of TIVADs.

\section{Materials and methods}

A total of 79 consecutive patients with unresectable or recurrent colorectal carcinomas underwent TIVAD placement surgeries between June 2007 and October 2008.

The patients were brought to the operating room and placed in a supine position. The operation was performed using local anesthesia ( $0.5 \%$ of xylocaine; AstraZeneca, UK). Patients who felt uneasy during the operation were administered an intravenous injection of $15 \mathrm{mg}$ of pentazocine (Astellas, Japan). Surgery was performed under maximal barrier precaution. The TIVAD placements were performed by two surgeons. Each stage of the surgical procedure of this study was supervised by one of the two surgeons.

The cephalic vein passes through the clavipectoral (deltopectoral) triangle to join the axillary vein. A $3-\mathrm{cm}$ wide skin incision was made in the infraclavicular region between the pectoralis major muscle and the triangular muscle (Fig. 1). The cephalic vein was identified in the adipose tissue along the deltopectoral groove. An incision of $3 \mathrm{~mm}$ in length was made on the surface of the vein. A Groshong catheter was inserted via the cephalic vein and connected to the port (BardPort X-port isp; Bard Access Systems Inc., Salt Lake City, UT, USA). The port was implanted in the subcutaneous space. The position of the catheter tip and the shape of the catheter lumen were confirmed by X-ray.

If the TIVAD placement by the CVCD approach was unsuccessful, the procedure was converted to the conventional percutaneous puncture approach of the subclavian vein. The operation time, and any intraoperative and post-operative complications were recorded.

\section{Results}

A total of 79 TIVAD placements were performed for 79 consecutive patients. TIVAD placement via the CVCD approach was completed in 74 patients (93.7\%). The CVCD 


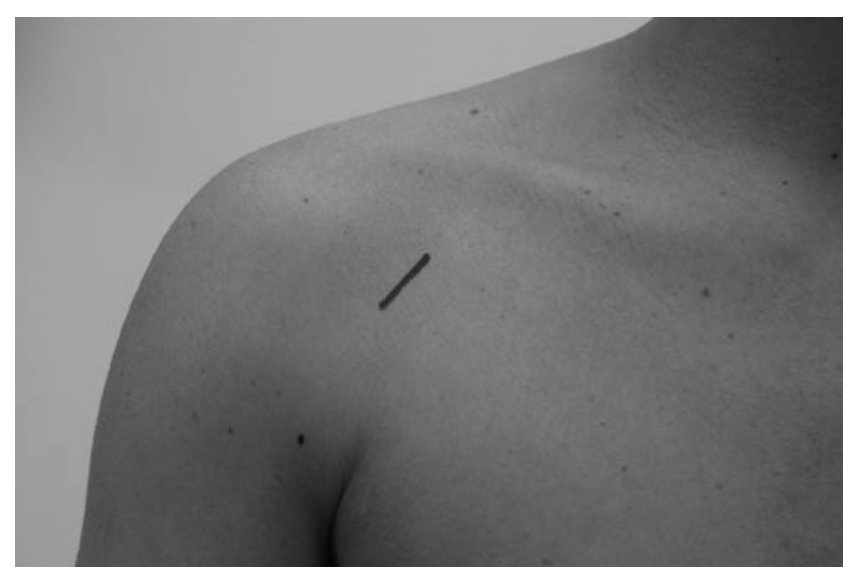

Figure 1. A skin incision site in the infraclavicular region.

approach was not successful in the remaining 5 patients $(6.3 \%)$. Subsequently, the 5 patients required conversion to a percutaneous puncture approach. The mean operation time was $34.7 \mathrm{~min}$ (median 30, range 17-103 $\mathrm{min}$ ). The cephalic vein was not detected in 2 cases, and in 1 case the cephalic vein was too narrow to insert the catheter. In 2 cases where the cephalic vein merged to the axillary vein, the tip of the catheter was not able to reach the superior vena cava. When the cephalic vein merged to the axillary vein vertically, the catheter tip reached only as far as the distal side of the axillary vein. In such cases, we attempted to change the merging angle of the cephalic and axillary veins to a more acute angle by leaning the head of the patient to the opposite side or moving the arm and shoulder. Manipulation of the angle normally allows the tip of the catheter to reach the superior vena cava when the merging point was vertical. However, in the 2 cases noted above, this manipulation was not successful.

No intraoperative or immediate postoperative complications were observed. A chest radiograph was obtained following the surgery to detect the position of the catheter tip and the distortion of the catheter lumen (Fig. 2). A distortion in the catheter may indicate pinch-off syndrome. In this study, no catheter distortion was detected.

\section{Discussion}

TIVADs are normally placed using the percutaneous subclavian vein approach. The percutaneous approach has occasionally caused intraoperative and postoperative complications, such as pneumothorax, arterial puncture, hemothorax or injury to the brachial plexus (1-5). The intraoperative complication rate of the percutaneous approach was reported to be $3-7 \%$ (1-5). The technique used for TIVAD implantation is now considered to be safer due to the use of image-guided navigation techniques, such as venous ultrasonography or venography. However, the risk of complications remains when the percutaneous puncture approach is used.

CVCD does not require risking a puncture and is associated with a very low rate of complications. A number of studies have compared the cut-down and percutaneous approaches and reported the superiority and safety of the cutdown approach compared to the conventional percutaneous method $(9,10)$.

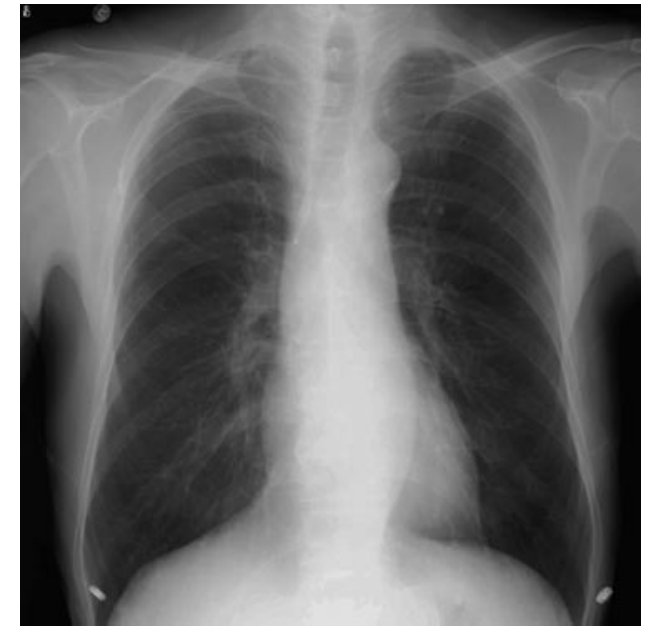

Figure 2. Chest X-ray following placement of a totally implantable venous access device.

Pinch-off syndrome has been reported as a complication of TIVAD $(12,13)$. This syndrome is thought to be caused by the compression of the catheter by the clavicle and the first rib $(12,13)$. Catheter compression may lead to obstruction followed by fracture of the catheter. Of note is that the pinch-off syndrome was not observed in a number of studies using the cut-down technique $(9,10,12)$.

Moreover, in the cardiovascular field, pinch-off syndrome is known as subclavian crush syndrome, which has been reported to occur with pacemaker leads implanted via the subclavian puncture technique. In these cases, conductor fracture and insulation breaches develop via compression of a lead that passes between the first rib and the clavicle. In this study, it was suggested that strong consideration should be given to obtain venous access primarily via the cephalic cut-down technique, due to the possibility of complications of subclavian crush syndrome with the percutaneous puncture approach.

The CVCD approach requires a surgical technique. Given the increase in the number of colorectal carcinoma patients worldwide, this technique may be of practical value. In this study, TIVAD placements were performed by the CVCD method and the outcome was evaluated.

The cephalic vein passes through the clavipectoral (deltopectoral) triangle to merge with the axillary vein. Since the cephalic vein shows few anatomical anomalies (11), it is suitable for cut-down and the placement of TIVAD.

TIVAD placement by CVCD was performed in 79 patients and completed in 74 patients. The remaining 5 patients required conversion to a percutaneous approach. The reasons for the conversion were due to abnormalities of the cephalic vein, including failure to detect the cephalic vein, a narrow cephalic vein and abnormal merging of the cephalic and axillary veins. In this study, the failure rate was found to be $6 \%$, which is lower than that in previous studies where a range of $8-30 \%$ was reported $(7,9,10)$.

Currently, the depth of the deltopectoral groove, location of cephalic vein and the margins of the axillary vein are routinely checked by ultrasonography prior to surgery. This increases the success rate of completing a CVCD method. 
We are therefore developing safer, more rapid and more practical placement methods for TIVADs.

In conclusion, this study showed that the CVCD technique is a safe and feasible approach for TIVAD placement. Additionally, this technique is associated with a lower rate of severe complications, reported to be up to $10 \%$, compared to the percutaneous method $(9,10)$.

\section{References}

1. Biffi $\mathrm{R}$, de Braud F, Orsi $\mathrm{F}$, et al: Totally implantable central venous access ports for long-term chemotherapy. A prospective study analyzing complications and costs of 333 devices with a minimum follow-up of 180 days. Ann Oncol 9: 767-773, 1998.

2. Kincaid EH, Davis PW, Chang MC, Fenstermaker JM and Pennell TC: 'Blind' placement of long-term central venous access devices: report of 589 consecutive procedures. Am Surg 65: 520-524, 1999.

3. Poorter RL, Lauw FN, Bemelman WA, Bakker PJ, Taat CW and Veenhof CH: Complications of an implantable venous access device (Port-a-Cath) during intermittent continuous infusion of chemotherapy. Eur J Cancer 32A: 2262-2266, 1996.

4. Nightingale CE, Norman A, Cunningham D, Young J, Webb A and Filshie J: A prospective analysis of 949 long-term central venous access catheters for ambulatory chemotherapy in patients with gastrointestinal malignancy. Eur J Cancer 33: 398-403, 1997.

5. Di Carlo I, Barbagallo F, Toro A, Sofia M, Lombardo R and Cordio S: External jugular vein cutdown approach, as a useful alternative, supports the choice of the cephalic vein for totally implantable access device placement. Ann Surg Oncol 12: 1-4, 2005.
6. Di Carlo I, Fisichella P, Russello D, Puleo S and Latteri F: Catheter fracture and cardiac migration: a rare complication of totally implantable venous devices. J Surg Oncol 73: 172-173, 2000.

7. Povoski SP: A prospective analysis of the cephalic vein cutdown approach for chronic indwelling central venous access in 100 consecutive cancer patients. Ann Surg Oncol 7: 496-502, 2000.

8. Di Carlo I, Cordio S, La Greca G, et al: Totally implantable venous access devices implanted surgically: a retrospective study on early and late complications. Arch Surg 136: 1050-1053, 2001.

9. Sarzo G, Finco C, Parise P, et al: Insertion of prolonged venous access device: a comparison between surgical cutdown and percutaneous techniques. Chir Ital 56: 437-442, 2004.

10. Chang HM, Hsieh CB, Hsieh HF, et al: An alternative technique for totally implantable central venous access devices. A retrospective study of 1311 cases. Eur J Surg Oncol 32: 90-93, 2006.

11. Le Saout J, Vallee B, Person H, Doutriaux M, Blanc J and Nguyen H: Anatomical basis for the surgical use of the cephalic vein (V. Cephalica). 74 anatomical dissections. 189 surgical dissections. J Chir 120: 131-134, 1983.

12. Andris DA, Krzywda EA, Schulte W, Ausman R and Quebbeman EJ: Pinch-off syndrome: a rare etiology for central venous catheter occlusion. J Parenter Enteral Nutr 18: 531-533, 1994.

13. Hinke DH, Zandt-Stastny DA, Goodman LR, Quebbeman EJ, Krzywda EA and Andris DA: Pinch-off syndrome: a complication of implantable subclavian venous access devices. Radiology 177: 353-356, 1990.

14. Roelke M, O'Nunain SS, Osswald S, Garan H, Harthorne JW and Ruskin JN: Subclavian crush syndrome complicating transvenous cardioverter defibrillator systems. Pace 18: 973-979, 1995. 